
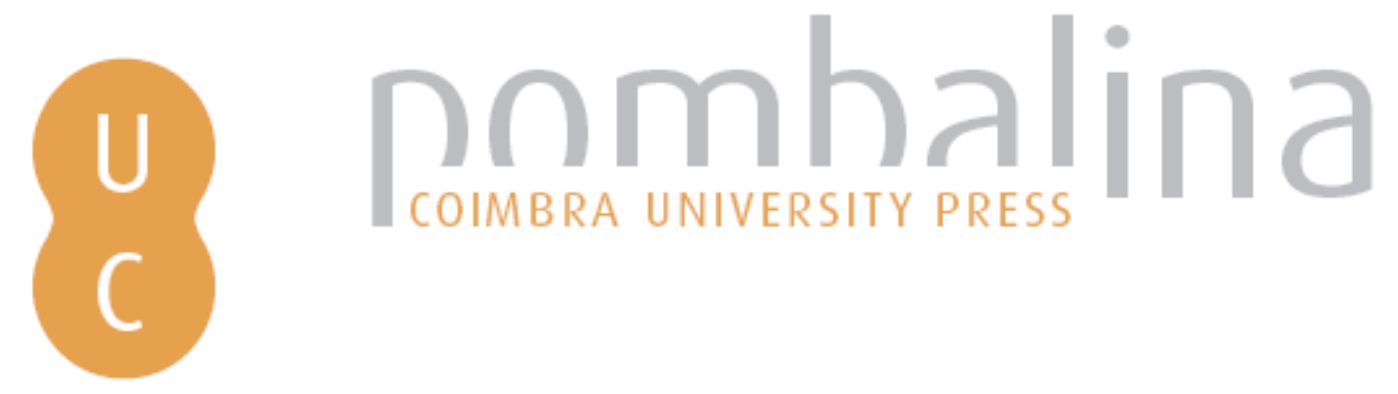

\title{
Estudo preliminar para reciclagem de resíduos de construção e demolição finos com adição de cal hidráulica natural
}
Autor(es):
Antunes, A. C. H.; Catarino, L.; Figueiredo, F.
Publicado por: Imprensa da Universidade de Coimbra
URL
persistente:
URI:http://hdl.handle.net/10316.2/31480
DOI:
DOI:http://dx.doi.org/10.14195/978-989-26-0531-9_38
Accessed : $\quad$ 26-Apr-2023 12:24:05

A navegação consulta e descarregamento dos títulos inseridos nas Bibliotecas Digitais UC Digitalis, UC Pombalina e UC Impactum, pressupõem a aceitação plena e sem reservas dos Termos e Condições de Uso destas Bibliotecas Digitais, disponíveis em https://digitalis.uc.pt/pt-pt/termos.

Conforme exposto nos referidos Termos e Condições de Uso, o descarregamento de títulos de acesso restrito requer uma licença válida de autorização devendo o utilizador aceder ao(s) documento(s) a partir de um endereço de IP da instituição detentora da supramencionada licença.

Ao utilizador é apenas permitido o descarregamento para uso pessoal, pelo que o emprego do(s) título(s) descarregado(s) para outro fim, designadamente comercial, carece de autorização do respetivo autor ou editor da obra.

Na medida em que todas as obras da UC Digitalis se encontram protegidas pelo Código do Direito de Autor e Direitos Conexos e demais legislação aplicável, toda a cópia, parcial ou total, deste documento, nos casos em que é legalmente admitida, deverá conter ou fazer-se acompanhar por este aviso.

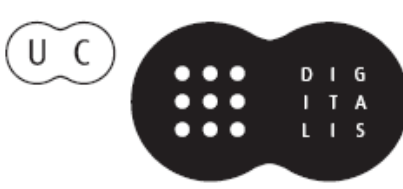





\title{
ESTUDO PRELIMINAR PARA RECICLAGEM DE RESÍDUOS DE CONSTRUÇÃO E DEMOLIÇÃO FINOS COM ADIÇÃO DE CAL HIDRÁULICA NATURAL
}

\author{
PRELIMINARY STUDY OF RECYCLING OF CONSTRUCTION \\ AND DEMOLITION WASTE WITH ADDITION OF NATURAL \\ HYDRAULIC LIME
}

A. C. H. Antunes ${ }^{1}$, L. Catarino ${ }^{2} \&$ F. Figueiredo ${ }^{3}$

\begin{abstract}
Resumo - A necessidade de evolução da civilização traz consigo várias descobertas e soluçóes, mas também gera problemas graves para o ambiente e, consequentemente, para o ser humano. A construção sustentável é neste momento uma emergência e, consequentemente, a reutilização e a reciclagem dos Resíduos de Construção e Demolição (RCD) uma necessidade extrema. Neste artigo, pretende-se estudar os RCD recolhidos num aterro de resíduos inertes da zona centro de Portugal, de forma a avaliar as suas características e possíveis aplicaçóes. Este artigo tem como objetivo contribuir para o sucesso da reciclagem dos RCD. O estudo dos RCD efetuado evidencia a sua previsível grande heterogeneidade. Os provetes obtidos por adição aos RCD de $10 \%$ de cal hidráulica natural, utilizada como ligante, apresentam uma baixa resistência à compressão uniaxial $(0,08-0,5 \mathrm{MPa})$, mas uma possível compatibilidade da sua utilização em construçóes de terra.
\end{abstract}

Palavras-chave - RCD, construção sustentável, aterro, reciclagem, ambiente

Abstract - Although progress carries many discoveries and solutions, it also has negative effects to the environment, and consequently to human beings. Nowadays, sustainable construction is seriously required, and the Construction and Demolition Waste (CDW) recycling is a huge priority. This paper is a preliminary study aimed for improving the recycling of the

\footnotetext{
${ }^{1}$ Centro de Geociências, Departamento de Ciências da Terra, Universidade de Coimbra, Largo Marques de Pombal 3000-272 Coimbra.

${ }^{1}$ celia.antunes@gmail.com

2 lidiagil@dct.uc.pt

3 fpedro@dct.uc.pt
} 
$C D W$. The main goal is to analyze the construction and demolition waste coming from a landfill in central Portugal, assessing their properties and potential applications. The CDW study carried out and described in this work confirms the waste heterogeneity. The uniaxial compressive strength obtained, in test specimen made with 10\% of natural hydraulic lime used as binder, is low (0.08-0.5MPa) but compatible with the use of this material in earth construction.

Keywords - CDW, sustainable construction, landfill, recycling, environment

\section{1 - Introduçáo}

A crescente evolução da população levou a um aumento exponencial do consumo de recursos naturais e energéticos, assim como do volume de resíduos sem utilização. Até às mais recentes décadas não existiam preocupaçôes na atividade da construção civil que incluíssem a gestão dos resíduos produzidos (NENO, 2010). Segundo Evangelista (2007), esta despreocupação na reutilização de resíduos provenientes da construção civil deve-se às preocupaçôes governamentais e/ou de fiscalização serem deficientes e permitirem a deposição deste tipo de resíduos em vazadouros ilegais, à relativa abundância de matéria-prima em Portugal e à fase precoce em que se encontram os conhecimentos científicos sobre os temas da reciclagem e reutilização dos Resíduos de Construção e Demolição (RCD).

Apesar de já se usar RCD na antiguidade, só em 1928 é que estes materiais começaram a ser estudados. O seu uso na Europa só se iniciou, de uma forma mais séria, depois da II Guerra Mundial, devido à necessidade de reconstrução de várias cidades que ficaram totalmente destruídas. Atualmente a Europa desperdiça cerca de 200 milhóes toneladas de resíduos de cimento e pedra por ano. Esta quantidade de material é suficiente para construir uma estrada de seis faixas entre Londres e Roma (MYMRIN \& CORREA, 2005).

A necessidade de implementar medidas de redução de detritos na indústria da construção é tâo ou mais importante que nas restantes indústrias, uma vez que a capacidade dos aterros é cada vez mais escassa, sendo necessário integrar métodos alternativos de deposição e tratamento dos detritos. Dado que a existência de aterros controlados pressupóe o uso do solo como recurso e comporta custos inerentes bastante elevados, a reciclagem poderá contribuir para a redução destes problemas, minimizando as quantidades de materiais depositados e reduzindo, em paralelo, a despesa de deposição. Desta minimização resultam não só redução de custos como também a redução do volume de extração de matérias-primas, garantindo a preservaçáo de recursos naturais limitados. Por outro lado, quer a incorporação de resíduos na produção de materiais, quer a reutilização de materiais traduzem-se numa poupança energética e diminuição da contaminação atmosférica (SALINAS, 2002).

A quantidade de RCD produzida na UE é de cerca de 33\% do total dos resíduos (KAZMIERCZYK \& REICHEL, 2010). Estes têm um grande potencial para serem reutilizados, sendo apenas necessário a criação de condiçóes adequadas para lidar com o problema de deposição de RCD, ou seja, aumentar o tempo de vida destes resíduos, possibilitando uma melhor gestão de recursos naturais e, consequentemente, reduzir o impacte ambiental. Estas razóes levaram a Comissão Europeia a apontar os RCD como uma prioridade. A diretiva 2008/98/CE (Waste Framework Directive - WFD) requer que os estados membros da UE tomem as medidas necessárias de forma a atingir um mínimo de 70\%, em massa, de RCD preparados para reutilizar, reciclar e outros fins até 2020 (MONIER et al., 2011). 
Os RCD necessitam de uma solução adequada para se obter uma construção sustentável em Portugal. Como tal, deverão pôr-se em prática estratégias que possam prevenir a sua produção, como também avaliar o ciclo de vida dos materiais aquando a sua utilização, minimizando as quantidades produzidas por meio da redução, reutilização e reciclagem até ao seu destino final (BARANDAS, 2009).

As preocupaçóes ambientais com que se confronta a sociedade atual fazem com que seja urgente repensar as atuais tecnologias construtivas tornando imperativas novas e mais sustentáveis formas de habitação (TORGAL \& JALALI, 2010).

Tendo como base a hipótese, por nós formulada, de que os RCD podem ser utilizados na construção de blocos semelhantes aos de adobe, adquirindo assim semelhanças com as construçóes em terra, decidimos realizar alguns testes. Para isso foi necessária a escolha de um ligante que respondesse às necessidades dos RCD. O ligante escolhido neste estudo inicial foi a cal hidráulica natural, devido às suas características físicas, químicas e mecânicas compatíveis com a heterogeneidade dos RCD recolhidos e ainda a capacidade de adquirir presa na presença de água.

Este trabalho pretende contribuir para o desenvolvimento da investigaçáo existente no domínio da gestão dos Resíduos de Construção e Demolição, através do estudo de formas alternativas da utilização deste material. Neste sentido, espera-se que este estudo auxilie na sustentabilidade da indústria da construção, uma vez que os resíduos por ela gerados são, nos últimos anos, uma preocupação a nível nacional e internacional. Assim sendo, será estudada a hipótese da produção de material de construção a baixo custo através da exploração das potencialidades dos resíduos, nomeadamente, aplicação dos RCD com dimensôes inferiores a $4,0 \mathrm{~mm}$, sem qualquer tratamento prévio ou diferenciação dos vários componentes.

Para que seja possível fundamentar este trabalho, foram analisadas diferentes amostras recolhidas em vários locais do aterro de resíduos inertes de Portunhos, e foi avaliado o comportamento mecânico deste material quando misturado com $10 \%$ de cal hidráulica natural.

\section{2 - Métodos experimentais}

Foram recolhidas quatro amostras (Fig. 1) em diferentes locais do aterro de resíduos inertes, situado em Portunhos, Cantanhede.
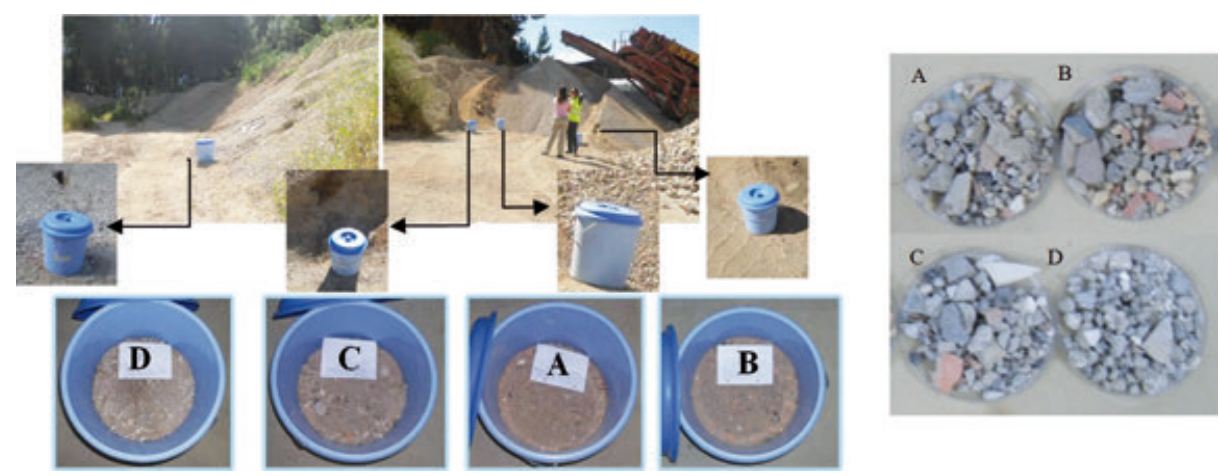

Fig. 1 - Amostras recolhidas no aterro de resíduos inertes de Portunhos. 
O procedimento experimental foi dividido em duas fases. Inicialmente procedeu-se à caracterização das amostras de RCD recolhidas no aterro (amostras $\mathrm{A}, \mathrm{B}, \mathrm{C}, \mathrm{D}$ ) e na segunda fase definiram-se seis grupos de provetes (grupos PG, PH, PI, PJ, PL, PM), três destes constituídos pela mistura das amostras A e B (PH, PJ, PM) e os restantes pela mistura das amostras C e D (PG, PI, PL), que foram submetidos ao ensaio de ultrassons e ao ensaio de resistência à compressão uniaxial.

Após classificação granulométrica constatou-se que a fração inferior a $4,0 \mathrm{~mm}$ constituía a maioria da amostra pelo que foi decidido utilizar apenas essa fração para os ensaios a efetuar.

Os ensaios realizados às amostras de RCD foram a análise granulométrica (método por peneiração e granulometria a laser) e a análise mineralógica por difração de raios $\mathrm{X}$ de lâmina sedimentada, para além da sua observação macroscópica e ao microscópio estereográfico.

$\mathrm{Na}$ segunda fase deste trabalho realizaram-se grupos de provetes prismáticos (4,0x4,0x16,0 cm) constituídos por conjuntos de amostras de RCD, denominados por PG, PH, PI, PJ, PL, PM (Fig. 2). Cada grupo é constituído por seis provetes cuja obtenção obedeceu às mesmas condiçóes.
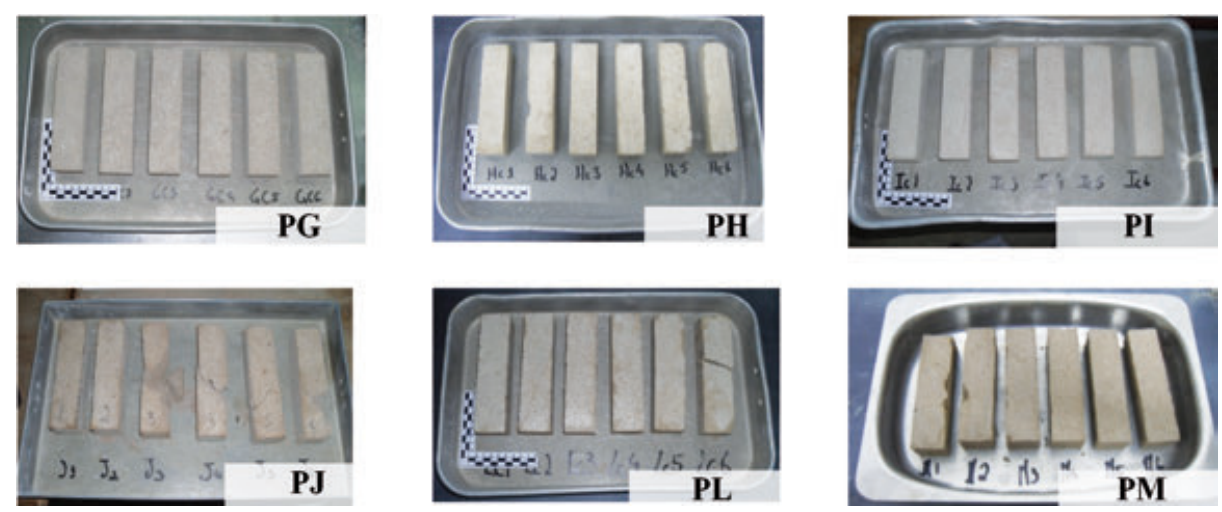

Fig. 2 - Grupo de seis provetes prismáticos $(4,0 \times 4,0 \times 16,0 \mathrm{~cm})$ de RCD com cal hidráulica natural.

Os provetes foram preparados com a fração das amostras de RCD inferior a $4,0 \mathrm{~mm}, 10 \%$ de cal hidráulica natural e misturados com água de maneira a obter uma mistura trabalhável.

Foram realizados grupos de provetes com dois tipos de amostra diferentes e dois tipos de cura (Fig. 3). 


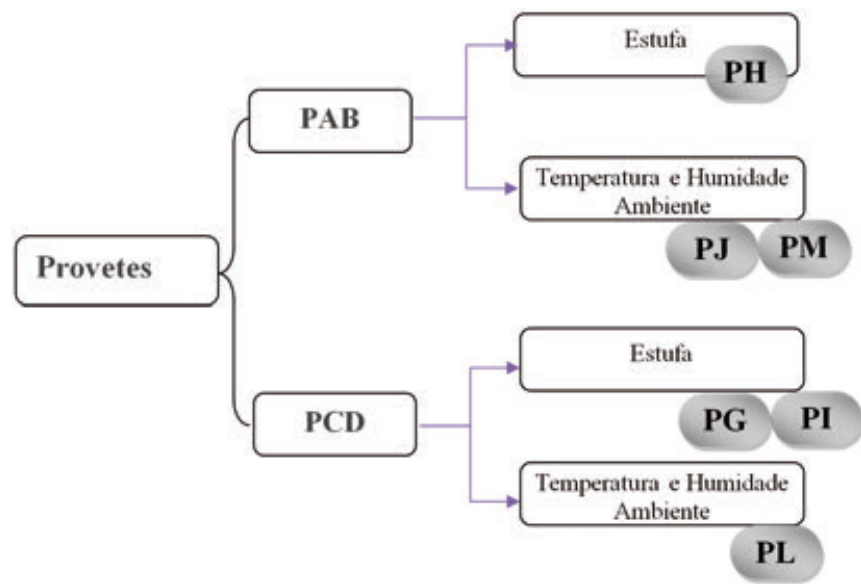

Fig. 3 - Esquema dos diferentes grupos de provetes (PAB - provetes realizados com a junçăo das amostras A e B (grupos PH, PJ e PM); PCD - provetes realizados com a junção das amostras C e D (grupos PG, PI e PL)).

Foram efetuados ensaios aos provetes dos diferentes grupos: o ensaio de ultrassons (Fig. 4) para determinar o módulo de Young e o ensaio de resistência à compressão uniaxial (Fig. 5).

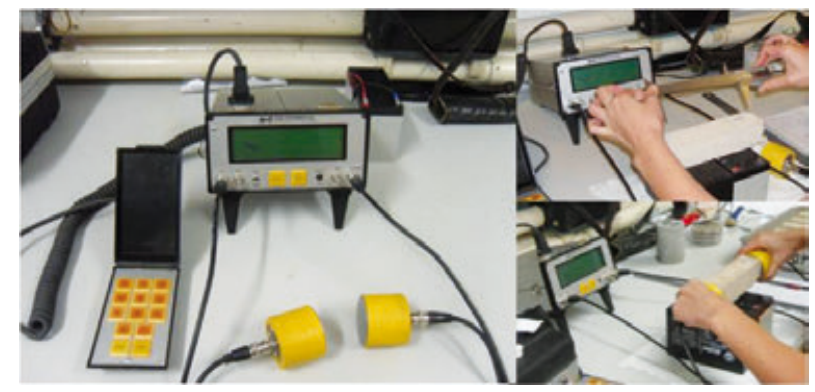

Fig. 4 - Equipamento V-Meter MarkII, utilizado para medir a velocidade das ondas de compressão.

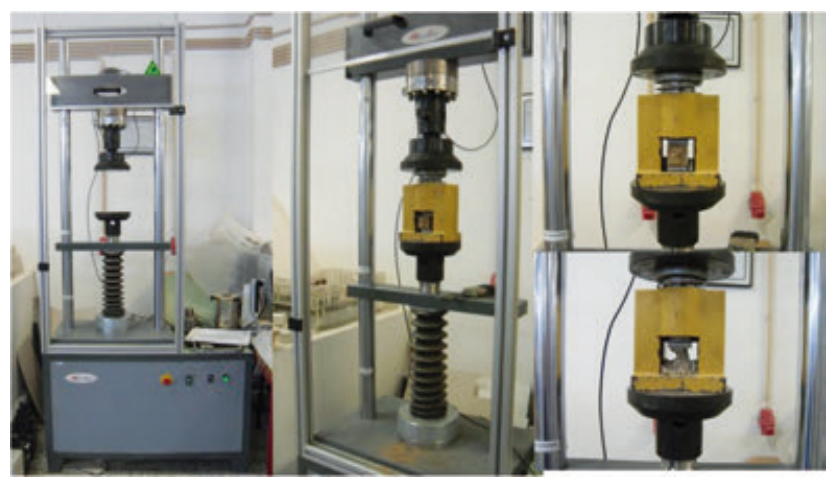

Fig. 5 - Equipamento MicroTest modelo $\mathrm{PB} / 20$ onde foi realizado o ensaio de resistência à compressão uniaxial. 
A velocidade de propagação de ultrassons nos provetes prismáticos foi avaliada de forma direta, através do posicionamento dos transdutores nas extremidades dos provetes, e no registo da velocidade das ondas de compressão (ou ondas $\mathrm{P}$ ) que percorreram os provetes com uma extensão longitudinal de $16 \mathrm{~cm}$. Após a recolha dos valores da velocidade das ondas P foi calculado o Módulo de Young (equaçáo1).

(1)

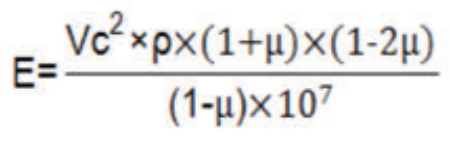

onde,

$\mathrm{E}=$ Módulo de Young (MPa);

$V c^{2}=$ Quadrado da velocidade

da onda de compressão (onda p)

$(\mathrm{cm} / \mathrm{s})$;

$\rho=$ Densidade $\left(\mathrm{g} / \mathrm{cm}^{3}\right)$

$\mu=$ Coeficiente de Poisson

O ensaio de determinação da resistência à compressão uniaxial foi realizado sobre meios provetes $(4,0 \times 4,0 \times 8,0 \mathrm{~cm})$, centrados entre os pratos inferior e superior do equipamento. De seguida aplicaram-se forças gradualmente crescentes até que se atingisse o ponto de rotura, registando o respetivo valor da força.

A tensão de rotura à compressão uniaxial é dada pelo quociente entre a carga máxima e a área da secção transversal onde foi aplicada a força (equação 2).

onde,

$\sigma_{\text {compressão }}=$ Tensão de rotura à compressão uniaxial (MPa);

(2)

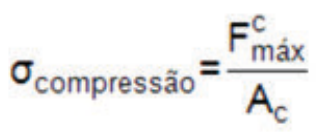

$\mathrm{F}_{\text {máx }}^{\mathrm{c}}=$ Carga máxima $(\mathrm{N})$;

$A_{c}=$ Área da secção transversal

onde foi aplicada e força

$\left(\mathrm{mm}^{2}\right)$.

\section{3 - Discussão de resultados}

\section{1 - Caracterização das amostras de RCD recolhidas}

Através da observação das curvas granulométricas (Fig. 6) das diferentes amostras, constata-se que, a nível granulométrico, as amostras A e B são semelhantes e o mesmo acontece com as amostras C e D. 


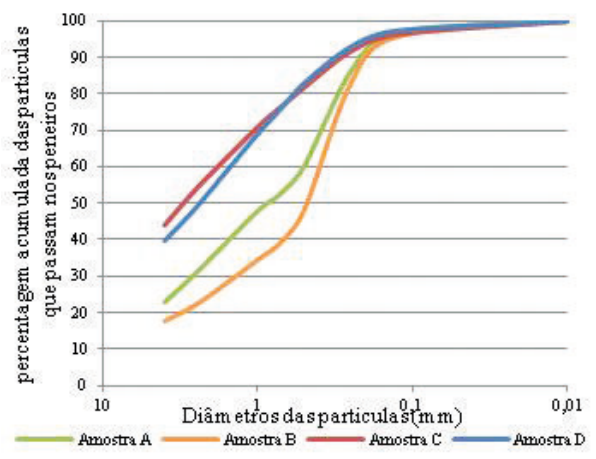

A

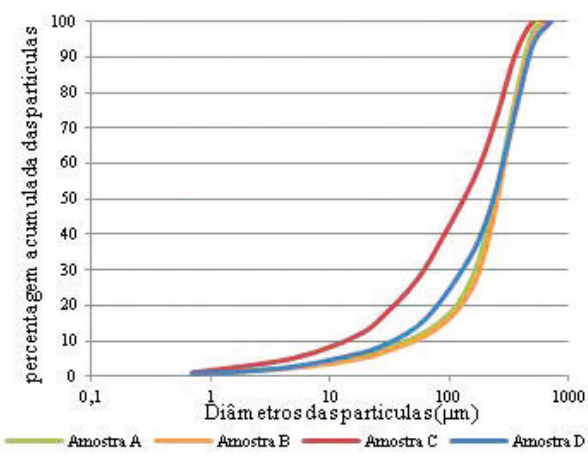

$\mathrm{B}$

Fig. 6 - Curvas granulométricas. A - Método de peneiração, B - Granulometria a laser.

Nos diferentes difractogramas (Fig. 7) podemos observar a constituição mineralógica principal das amostras. Embora os resultados sejam avaliaçóes qualitativas é possível verificar que as amostras A e B contêm maior quantidade de quartzo e de moscovite do que de calcite, enquanto as amostras $\mathrm{C}$ e $\mathrm{D}$ têm quantidades idênticas destes dois minerais. Todas as amostras têm na sua composição calcite, quartzo, caulinite, moscovite, ilite e feldspato potássico (feldspato K), feldspato sódico-cálcico (feldspato $\mathrm{Na}-\mathrm{Ca}$ ). A amostra $\mathrm{C}$ contém também vestígios de gesso. Os difractogramas de todas as amostras são bastante semelhantes, sendo as maiores intensidades correspondentes ao mesmo tipo de minerais, quartzo, moscovite e calcite.

Verificou-se que as amostras recolhidas eram semelhantes duas a duas (A e B, C e D), em relação à sua tonalidade, à sua constituição e às curvas granulométricas. Por essa razão, para a elaboração dos provetes decidiu-se juntar as amostras duas a duas e formar dois grupos de provetes distintos em relação à sua constituição.

Pela análise feita às amostras suspeita-se que duas delas (amostras A e B) sejam originárias da demolição de construçóes com terra, pois estas tinham uma tonalidade amarelada/acastanhada e na sua constituição, verificada macroscopicamente, tinham grande quantidade de adobe, e consequentemente, maiores quantidades de argila. As outras duas amostras tinham uma tonalidade acinzentada, e na sua constituição tinham grande quantidade de betão e tijolo, e náo se observou adobe, suspeitando-se então, que estas duas últimas sejam originárias de construçôes mais recentes efetuadas com betão e tijolo. 

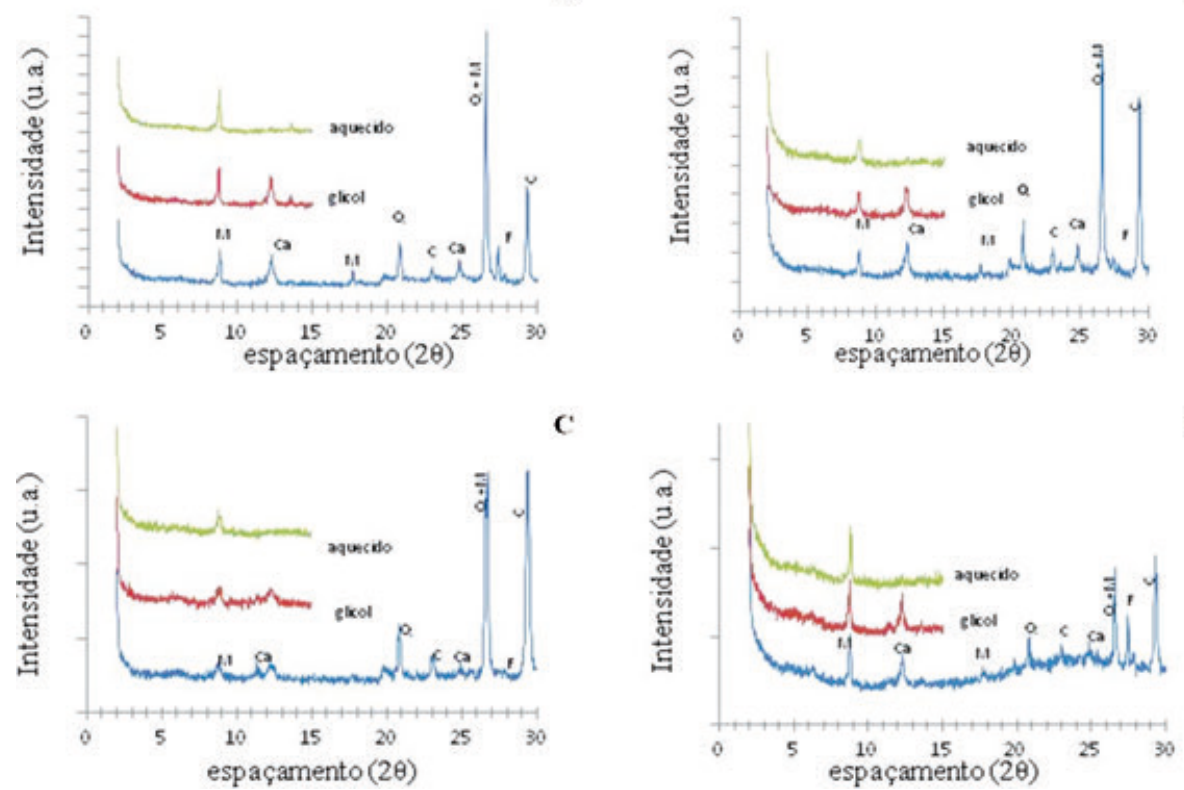

Fig. 7 - Difractogramas das diferentes amostras (C-Calcite, Ca-Caulinite, F-Feldspato, M-Moscovite,

Q-Quartzo).

\section{2 - Análise dos provetes}

Após a realização dos ensaios de caracterização dos provetes, verificou-se que as amostras que apresentavam maior quantidade de betão (amostras C e D), são mais resistentes que as amostras que possuem maiores quantidades de argila (Tabela 1).

Tabela 1 - Propriedades dos diferentes grupos de provetes.

\begin{tabular}{|c|c|c|c|c|c|c|}
\hline $\begin{array}{c}\text { Amostras de } \\
\text { RCD }\end{array}$ & & $\mathrm{CL}$ & & & $\mathrm{AB}$ & \\
\hline $\begin{array}{c}\text { Grupos de } \\
\text { provetes }\end{array}$ & PG & PI & PL & $\mathrm{PH}$ & PJ & PM \\
\hline Tipo de cura & $\begin{array}{l}\text { Estufa } \\
40^{\circ} \mathrm{C}\end{array}$ & $\begin{array}{l}\text { Estufa } \\
40^{\circ} \mathrm{C}\end{array}$ & $\begin{array}{l}55 \%-65 \% \text { de } \\
\text { humidade; } \\
19^{\circ}-23^{\circ} \mathrm{C} \text { de } \\
\text { temperatura }\end{array}$ & $\begin{array}{l}\text { Estufa } \\
40^{\circ} \mathrm{C}\end{array}$ & $\begin{array}{l}55 \%-65 \% \text { de } \\
\text { humidade; } \\
19^{\circ}-23^{\circ} \mathrm{C} \text { de } \\
\text { temperatura }\end{array}$ & $\begin{array}{l}55 \%-65 \% \text { de } \\
\text { humidade; } \\
19^{\circ}-23^{\circ} \mathrm{C} \mathrm{de} \\
\text { temperatura }\end{array}$ \\
\hline $\begin{array}{l}\text { Tempo de cura } \\
\text { (dias) }\end{array}$ & 41 & 27 & 20 & 36 & 20 & 11 \\
\hline $\begin{array}{l}\text { Módulo de } \\
\text { Young (MPa) }\end{array}$ & 1081,8 & 743,7 & 1155,4 & 349,5 & 552,1 & 458,7 \\
\hline $\begin{array}{l}\text { Tensáo de rotura } \\
\text { à compressáo } \\
(\mathrm{MPa})\end{array}$ & 0,472 & 0,241 & 0,366 & 0,084 & 0,083 & 0,120 \\
\hline
\end{tabular}


Verificou-se ainda que as amostras que estiveram a secar nas condiçóes de temperatura e humidade ambiente (temperatura de $19-23^{\circ} \mathrm{C}$ e a humidade entre $55 \%-65 \%$ ) apresentam módulos de elasticidade e tensôes de rotura à compressão maiores que as amostras que estiveram na estufa a aproximadamente $40^{\circ} \mathrm{C}$, exceto o grupo de provetes $\mathrm{PG}$, que apresentou os maiores valores de tensão de rotura à compressão e esteve à temperatura de $40^{\circ} \mathrm{C}$ na estufa. Estes resultados poderão justificar-se com a quantidade de água adicionada na preparação dos provetes $\mathrm{PG}$, que foi inferior à dos restantes grupos de provetes, com a compactação que sofreram e ao tempo de cura, que foi superior ao dos restantes grupos de provetes.

\section{4 - Conclusão}

Com a pesquisa efetuada sobre os RCD para a elaboração deste trabalho, ficou patente a urgência de valorizar e utilizar este tipo de resíduos.

Perante todas as razóes apresentadas neste artigo e com base nos resultados obtidos é possível concluir que, apesar dos baixos valores obtidos nos ensaios de resistência à compressão uniaxial que foram realizados às amostras de RCD $(0,08-0,5 \mathrm{MPa})$, o estudo de aplicaçóes para este material é, de facto, muito importante e acredita-se que estes resíduos têm um elevado potencial de utilização na indústria de construção, podendo para isso proceder-se à modificação dos ligantes e dos teores de água utilizados.

Agradecimentos - À empresa J. Batista Carvalho - Aterro de Resíduos Inertes pela cedência dos materiais necessários, em particular à Eng. ${ }^{a}$ Liliana Monteiro pela disponibilidade prestada e aos Eng. Silvino Capitão e Jorge Lourenço, e ao Sr. António Amaral (ISEC), pela disponibilidade e pelo apoio técnico na fase experimental. Este trabalho foi financiado pelo Estado Português através da FCT - Fundação para a Ciência e a Tecnologia no âmbito do projecto PEst-OE/CTE/UI0073/2011 do Centro de Geociências.

\section{Referências Bibliográficas}

BARANDAS, R. (2009) - Gestão de Residuos de Construção e Demolição. Engenharia Civil, Universidade de Trás-os-Montes e Alto Douro. Vila Real - Portugal: s.n. p 1-96, Dissertação de mestrado

EVANGELISTA, L. (2007) - Betóes executados com agregados finos reciclados de betão. Engenharia Civil, Instituto Superior Técnico - Universidade Técnica de Lisboa. Lisboa: s.n. p. 1-178, Dissertação de mestrado.

KAZMIERCZYK, P. e REICHEL, A. (2010) - The European Environment. State And Outlook 2010. Material Resources And Waste. European Environment Agency, Copenhagen, 2010. p. 1-46.

MONIER, V., MUDGAL, S.; HESTIN, M.; TRARIEUX, M. e MIMID, S. (2011) - Service Contract on Management of Construction and Demolition Waste - SR1. Paris: Bio Intelligence Service. p. 1-240.

MYMRIN, V. e CORREA, S. M. (2005) - New construction from concrete production and demolotion wastes and lime production waste. Construction and Building Materials, 21, p. 578-582.

NENO, C. (2010) - Desempenho de argamassas com incorporação de agregados finos provenientes da trituração do betão. Engenharia Civil, Instituto Superior Técnico - Universidade Técnica de Lisboa. Lisboa: s.n. p. 1-128, Dissertação de mestrado.

SALINAS, L. (2002) - Gestão de resíduos de construção e demolição. Engenharia Civil, Faculdade de Ciências e Tecnologia - Universidade de Coimbra. Coimbra: s.n. p. 1-273, Dissertaçáo de mestrado

TORGAL, F. e JALALI, S. (2010) - Ensaios de avaliação da durabilidade das construçôes em terra. Terra em seminário 2010. 90 Seminário Ibero-Americano de Arquitectura e Construção com terra. Ed. Maria Fernandes, Mariana Correia, Filipe Jorge. Argumentum, p. 138-141 